NIST

PUBLICATIONS

\title{
A Boussinesq Algorithm for Enclosed Buoyant Convection in Two Dimensions
}

R. G. Rehm

H. C. Tang

H. R. Baum

J. S. Sims

D. M. Corley

U.S. DEPARTMENT OF COMMERCE Natlonal Institute of Standards and Tochnology

Computing and Applled Mathematics Laboratory

Galthorsburg, MD 20899 



\section{A Boussinesq Algorithm for Enclosed Buoyant Convection in Two Dimensions}

R. G. Rehm

H. C. Tang

H. R. Baum

J. S. Sims

D. M. Corley

U.S. DEPARTMENT OF COMMERCE Natlonal Institute of Standards and Technology

Computing and Applled Mathematics Laboratory

Galthorsburs, MD 20899

March 1991

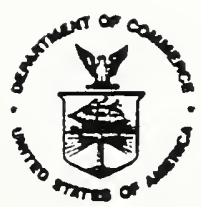

US. DEPARTMENT OF COMMERCE Robert A. Mosbacher, Secretary NATONAL INBTIUUTE OF STANDARDS AND TECKNOLOGY John W. Lyone, Director 



\title{
A Boussinesq Algorithm for Enclosed Buoyant Convection in Two Dimensions
}

\author{
R.G. Rehm, H.C. Tang, H.R. Baum, J.S. Sims and D.M. Corley \\ National Institute of Standards and Technology \\ Gaithersburg, MD 20899
}

Approximate equations for a Boussinesq model with viscous dissipation and thermal conduction describing buoyant convection driven by a heat source in a rectangular enclosure are derived. The finite-difference algorithm for computing transient solutions in two dimensions to these equations is presented. The algorithm allows the enclosure fluid to be stratified in a direction parallel to the enclosure walls initially, or for gravity to have an arbitrary direction relative to the enclosure (but with no initial stratification). Computational results of transient, two-dimensional buoyant convection for very high resolution are presented. The hydrodynamics is directly based on the time-dependent Navier-Stokes equations; the model is valid in the Boussinesq approximation. No turbulence model or other empirical parameters are introduced. There is no inflow or outllow at boundaries; this assumption, although rather restrictive, allows the mathematical problem to be properly formulated so that no other empiricism is introduced by specification of the algorithmic boundary conditions. A finite-difference scheme second-order in space and first-order in time is used to integrate the evolution equations, and an elliptic solver is used to solve the pressure equation. The algorithms have been verified by comparisons with exact solutions to the equations in simple, special cases, and predictions of the overall model when the viscosity and thermal cond uctivity are zero have been compared with experimental results. The use of Lagrangian particle tracking allows one to visualize the flow patterns.

\section{Introduction}

The authors have published previously a description of the mathematical model and their algorithm for computation of the buoyant convection in- 
duced by a fire evolving in a room [1] - [7]. The model is for a dissipation free, thermally expandable fluid, i.e., one for which density and temperature variations can be large, but pressure variations are small [1], and for a Boussinesq model, one in which the density variations are also small. The algorithm can be generalized easily to include viscous dissipation and thermal conductivity. Previously the authors had argued that one could not resolve both large-scale motions associated with room-scale buoyant convection and motions at the dissipative length scale with computational resources available. However, with the revolution in computational capability currently taking place, this statement is no longer strictly true, at least in two dimensions.

In this document the authors generalize their model and algorithm in two dimensions to include viscous dissipation and thermal conduction. Also included are recently introduced generalizations which allow for either an initial background stratification in a direction parallel to the enclosure walls or for the enclosure to have an arbitrary direction relative to gravity (but not both in the same calculation). We present the details of the algorithm in this report (which may be regarded as a working document on the two-dimensional boussinesq code with dissipation). Because the model is restricted to two dimensions, very high resolution computations can be performed, which allow us to resolve both large-scale buoyant convection and small scale dissipation for Reynolds numbers of interest for enclosure fires. The model of buoyant convection is presented in Section 2 and a brief description of the numerical methods in Section 3. Section 4 contains a detailed description of the algorithm. Some sample results, together with discussion of the physical phenomena observed, are described in Section 5. An Appendix is also included which contains the algorithm used to introduce and track the Lagrangian particles.

\section{Hydrodynamic Model}

Traditionally, two approaches to the computation of fire-induced buoyant convection have been reported: direct integration of the Navier-Stokes equations using molecular values for viscosity and thermal conductivity or integration of these equations using a turbulent viscosity and conductivity to account for fluctuations occurring at the large Reynolds numbers of 
practical interest. The former approach, although most desirable, is not practically feasible in three dimensions with today's computers; computers are not big and fast enough to calculate simultaneously the largest scales of buoyant convection and the smallest scales at which dissipation occurs. Alternately, the use of a turbulence model in the equations introduces functional forms and empirical constants which do not have a fundamental theoretical basis at this time. However, in two dimensions, direct simulation of the Navier-Stokes equations at Reynolds numbers of practical interest for fire-driven flows is possible and even practical, and these simulations are the subject of this report.

The essence of the buoyant convection model is described as follows. We consider a Boussinesq fluid with constant viscosity and thermal conductivity in a rectangular enclosure driven by a prescribed heat source. We start with the equations of motion for a thermally expandable ideal gas [1] in which we include viscous dissipation and thermal conductivity (with constant viscous and conduction coefficients).

$$
\begin{aligned}
\frac{\partial \rho}{\partial t}+\frac{\partial}{\partial x_{i}}\left(\rho u_{i}\right) & =0 \\
\rho\left(\frac{\partial u_{i}}{\partial t}+u_{j} \frac{\partial u_{i}}{\partial x_{j}}\right)+\frac{\partial p}{\partial x_{i}}-\rho k_{i} g & =\rho \nu \frac{\partial^{2} u_{i}}{\partial x_{j}^{2}} \\
\rho C_{p}\left(\frac{\partial T}{\partial t}+u_{j} \frac{\partial T}{\partial x_{j}}\right) & =Q+K \frac{\partial^{2} T}{\partial x_{j}^{2}} \\
p_{0} & =\rho R T
\end{aligned}
$$

Here, all symbols have their usual fluid dynamical meaning: $\rho$ is density, $u_{i}$ are the velocity components, $p$ is pressure, $g$ is the acceleration of gravity, $k_{i}$ are the components of the vector describing the direction of the gravity vector, $\nu$ is kinematic viscosity, $C_{p}$ is the constant-pressure specific heat, $T$ is temperature and $K$ is the thermal conductivity, $t$ is time and $Q$ is the spatially and temporarly prescribed heat source.

If we combine these equations as described in [2], nondimensionalize them as described in the appendix of this reference and make the Boussinesq approximation, we obtain the following set of equations, 


$$
\begin{aligned}
\frac{\partial \rho}{\partial t}+u_{i} \frac{\partial \rho}{\partial x_{i}} & =-\frac{\gamma-1}{\gamma} Q+\kappa \nabla^{2} \rho \\
\frac{\partial u_{i}}{\partial t}+u_{j} \frac{\partial u_{i}}{\partial x_{j}}+\frac{\partial p}{\partial x_{i}}-\left(\rho-\rho_{0}(z)\right) k_{i} & =\nu \nabla^{2} u_{i} \\
\frac{\partial u_{i}}{\partial x_{i}} & =0
\end{aligned}
$$

Here, all symbols have the meanings given above but in dimensionless form, $\rho_{0}(z)$ is the initially stratified density profile assumed to depend only upon the vertical coordinate $z$, and $\kappa$ is the dimensionless thermal conductivity. The dimensionless quantities are defined as follows: lengths are relative to the height of the enclosure, pressure is relative to ambient pressure, time is relative to the height of the enclosure and the velocity scale, and velocity is relative to a scale $U$, and the density perturbation is relative to ambient density with a small parameter $\beta$. If we denote temporarily the dimensional quantities by an asterisk, then these relationships can be written as follows:

$$
\begin{aligned}
& x_{i}^{*}=H x_{i}, \quad t^{*}=\frac{H}{U} t \\
& u_{i}^{*}=U u_{i} \\
& p^{*}=\rho_{0}(0) U^{2} p \\
& \rho^{*}=\rho_{0}(0)(1+\beta \rho) \\
& \rho_{0}^{*}\left(z^{*}\right)=\rho_{0}(0)(1+\beta \rho(z))
\end{aligned}
$$

$U$ and $\beta$ are defined in terms of the magnitude of the heat source as follows:

$$
\begin{array}{r}
\beta=U^{2} /(g H) \\
U=\left(Q_{0} g /\left(\rho_{0} C_{p} T_{0} H\right)\right)^{1 / 3} \\
U=\left(q_{0} g /\left(\rho_{0} C_{p} T_{0}\right)\right)^{1 / 3}
\end{array}
$$

Here $Q_{0}$ is the strength of the three-dimensional heat source in units of energy per unit time, $q_{0}$ is the strength of the two-dimensional heat source in energy per unit length per unit time, $H$ is the height of the enclosure and all other quantities have their usual meanings. See [2] and [6] for more details of the scaling. Henceforth, all variables will be regarded as dimensionless. 
In the two-dimensional case, these equations can be rewritten as follows:

$$
\begin{aligned}
\frac{\partial \tilde{\rho}}{\partial t}+\tilde{F}_{\rho x}+\tilde{F}_{\rho z} & =-\frac{\gamma-1}{\gamma} Q+\kappa\left(\frac{\partial^{2} \tilde{\rho}}{\partial x^{2}}+\frac{\partial^{2} \tilde{\rho}}{\partial z^{2}}+\frac{\partial^{2} \rho_{0}}{\partial z^{2}}\right) \\
\frac{\partial u}{\partial t}+F_{x}+\frac{\partial p}{\partial x}-\tilde{\rho} g_{x} & =\nu\left(\frac{\partial^{2} u}{\partial x^{2}}+\frac{\partial^{2} u}{\partial z^{2}}\right) \\
\frac{\partial w}{\partial t}+F_{z}+\frac{\partial p}{\partial z}-\tilde{\rho} g_{z} & =\nu\left(\frac{\partial^{2} w}{\partial x^{2}}+\frac{\partial^{2} w}{\partial z^{2}}\right) \\
\frac{\partial u}{\partial x}+\frac{\partial w}{\partial z}=0 &
\end{aligned}
$$

where

$$
\begin{array}{r}
\tilde{\rho}=\rho-\rho_{0}(z) \\
\tilde{F}_{\rho x}=u \frac{\partial \rho}{\partial x} \\
\tilde{F}_{\rho z}=w\left(\frac{\partial \rho_{0}}{\partial z}+\frac{\partial \rho}{\partial z}\right) \\
F_{x}=(1 / 2) \frac{\partial q^{2}}{\partial x}-w \omega \\
F_{z}=(1 / 2) \frac{\partial q^{2}}{\partial z}+u \omega \\
\omega=\frac{\partial w}{\partial x}-\frac{\partial u}{\partial z} \\
q^{2}=u^{2}+w^{2}
\end{array}
$$

Boundary conditions for these equations are that there be no inflow or outflow at boundaries, that there be a no-slip or free-slip conditions at boundary walls, and that the walls are adiabatic or kept at a constant temperature. The initial conditions are that the fluid is quiescent.

\section{Numerical Methods}

Equations (1) are a mixed parabolic/elliptic system of partial differential equations; i.e., the equations for the density and for the velocity components are parabolic, whereas that for the pressure is elliptic. The incompressible 
equations of hydrodynamics are well known to have this mixed character. When there is no dissipation in these equations, it is important not to introduce any through the numerical scheme. Analytical studies of the ability of several candidate finite difference schemes to calculate internal gravity waves [3] led to the conclusion that methods of second order accuracy in space and time would be necessary; without dissipation, the scheme chosen is dispersive, but not dissipative. All time derivatives are replaced by central differences over twice the time step size (a leap-frog scheme) while all viscous and conduction terms are either differenced at the lagged time level in one case, or according to a DuFort-Frankel scheme in the other. Both schemes, the lagged-dissipation one and the DuFort-Frankel, have been used. Other terms in the evolution equations (the first two of Eqs. (1)) are in general evaluated at the mid level of the three level scheme.

The spatial grid is taken to be uniform in each of the two directions, although the mesh length may be different in each direction. Within each rectangular mesh cell, vector components are evaluated at the sides and scalar quantities at the center of the cell. The staggered grid permits central differences to second order accuracy for all linear operations. The nonlinear terms must be considered separately. The density evolution equation in continuous form is the mass conservation equation minus the expression for the velocity divergence. Each of these two equations is approximated by central differrences and then subtracted. The density at all faces is approximated by the mean of the density at the centers of adjacent cells. This procedure ensures global mass conservation as well as second order accuracy.

The momentum equation is differenced in the vector invariant form shown in Eqs. (1). This ensures nonlinear stability and complete compatibility between the "primitive variable" formulation presented here and a vorticity, stream-function formulation (in the dissipation-free case), see [4] for details.

The pressure equation is the discretized version of the time derivative of the third of Eqs. (1), using the central difference approximation to the divergence of the velocity and with the time difference of the velocity replaced using the discretized momentum equations. Mathematically, the calculation of the pressure requires the solution of an elliptic partial differential equation. For the Boussinesq model, the linear algebraic system arising from its discretization has constant coefficients and can be solved by a fast 
direct method, see [6] for details. The solution to the pressure equation constitutes the bulk of the numerical computation since the density and the velocity are updated explicitly once the pressure gradients are known.

Finally, stability of the computational scheme imposes a limit on the time step size relative to the spatial mesh sizes, see [2] and [3]. Also, accuracy of the computation is an important consideration, which has been examined in [2], [3] and [6], and verification that the numerical methods solve the partial differential equations, at least in special cases, has been addressed in these references. In addition, the basic features, such as the plume rise-time in a uniform density environment, have been compared with experimental results to verify the basic predictive capability of the dissipation-free model [5]. With such careful consideration of the basic model and numerical methodology, we have confidence in the predictions made by these computations, and feel justified in interpreting physical features arising in them.

\section{The Algorithm}

\subsection{The Density Equation}

We now write out the details of the algorithm. First, for the density equation, return to the full continuity equation (in dimensional form):

$$
\begin{array}{r}
\frac{\partial \rho}{\partial t}+\frac{\partial}{\partial x_{i}}\left(\rho u_{i}\right)=0 \\
\frac{\partial \rho}{\partial t}+u_{i} \frac{\partial \rho}{\partial x_{i}}+\rho \frac{\partial u_{i}}{\partial x_{i}}=0
\end{array}
$$

Use the nondimensionalization presented earlier

$$
\frac{\partial \tilde{\rho}}{\partial t}+u_{i} \frac{\partial\left(\tilde{\rho}_{0}+\tilde{\rho}\right)}{\partial x_{i}}+\left[1+\beta\left(\tilde{\rho}_{0}+\tilde{\rho}\right)\right] \frac{\partial u_{i}}{\partial x_{i}}=0
$$

where $\rho=1+\beta\left(\tilde{\rho}_{0}+\tilde{\rho}\right)$, where now all variables are dimensionless and where we have divided through by $\beta$. If we now formally allow $\beta \rightarrow 0$, then we recover the Boussinesq equation for the density.

$$
\frac{\partial \tilde{\rho}}{\partial t}+u_{i} \frac{\partial\left(\tilde{\rho}_{0}+\tilde{\rho}\right)}{\partial x_{i}}+\frac{\partial u_{i}}{\partial x_{i}}=0
$$


The same procedure used above for the partial differential equations (PDE) can now be applied to the finite difference equations (FDE). We desire to keep conservation form for the FDE as well as for the PDE. Hence,

$$
\begin{aligned}
& \frac{\partial \rho_{i k}}{\partial t}+\left[\frac{\rho_{i+1, k}+\rho_{i k}}{2} u_{i k}-\frac{\rho_{i k}+\rho_{i-1, k}}{2} u_{i-1, k}\right] \frac{1}{\delta x} \\
& +\left[\frac{\rho_{i, k+1}+\rho_{i k}}{2} w_{i k}-\frac{\rho_{i k}+\rho_{i, k-1}}{2} w_{i, k-1}\right] \frac{1}{\delta z}=0
\end{aligned}
$$

or, rewriting,

$$
\begin{aligned}
& \frac{\partial \rho_{i k}}{\partial t}+\frac{\rho_{i+1, k} u_{i k}}{2 \delta x}-\frac{\rho_{i-1, k} u_{i-1, k}}{2 \delta x}+\frac{\rho_{i k}}{2 \delta x}\left(u_{i k}-u_{i-1, k}\right) \\
& +\frac{\rho_{i, k+1} w_{i k}}{2 \delta z}-\frac{\rho_{i, k-1} w_{i, k-1}}{2 \delta z}+\frac{\rho_{i k}}{2 \delta z}\left(w_{i k}-w_{i, k-1}\right)=0
\end{aligned}
$$

Using the identity

$$
a c-b d=[(a+b)(c-d)+(a-b)(c+d)] / 2
$$

we find

$$
\begin{array}{r}
\frac{\partial \rho_{i k}}{\partial t}+\frac{1}{4 \delta x}\left(\rho_{i+1, k}+\rho_{i-1, k}\right)\left(u_{i k}-u_{i-1, k}\right) \\
+\frac{1}{4 \delta x}\left(\rho_{i+1, k}-\rho_{i-1, k}\right)\left(u_{i k}+u_{i-1, k}\right)+\frac{\rho_{i k}}{2 \delta x}\left(u_{i k}-u_{i-1, k}\right) \\
+\frac{1}{4 \delta z}\left(\rho_{i, k+1}+\rho_{i, k-1}\right)\left(w_{i k}-w_{i, k-1}\right) \\
+\frac{1}{4 \delta z}\left(\rho_{i, k+1}-\rho_{i, k-1}\right)\left(w_{i k}+w_{i, k-1}\right)+\frac{\rho_{i k}}{2 \delta z}\left(w_{i k}-w_{i, k-1}\right)=0
\end{array}
$$

or

$$
\frac{\partial \rho_{i k}}{\partial t}+F_{\rho x i k}+F_{\rho z i k}+(1 / 2) \rho_{i k} \Delta_{i k}=0
$$

where

$$
\begin{array}{r}
\Delta_{i k}=\left(\frac{\partial u_{j}}{\partial x_{j}}\right)_{i k}=\left[\frac{\gamma-1}{\gamma p_{0}}\left(Q-\frac{p_{0} K}{R \rho^{2}} \nabla^{2} \rho\right)\right]_{i k} \\
F_{\rho x i k}=\frac{1}{4 \delta x}\left(\rho_{i+1, k}+\rho_{i-1, k}\right)\left(u_{i, k}-u_{i-1, k}\right) \\
+\frac{1}{4 \delta x}\left(\rho_{i+1, k}-\rho_{i-1, k}\right)\left(u_{i, k}+u_{i-1, k}\right)
\end{array}
$$




$$
\begin{aligned}
F_{\rho z i k}= & \frac{1}{4 \delta z}\left(\rho_{i, k+1}+\rho_{i, k+1}\right)\left(w_{i, k}-w_{i, k-1}\right) \\
& +\frac{1}{4 \delta z}\left(\rho_{i, k+1}-\rho_{i, k-1}\right)\left(w_{i, k}+w_{i, k-1}\right)
\end{aligned}
$$

Finally, use the same nondimensionalization as cited above, using $\rho_{i k}=$ $1+\beta\left(\tilde{\rho}_{0, i k}+\tilde{\rho}_{i k}\right)$. Note that only $\rho_{i k}$ and $\tilde{\rho}_{i k}$ depend upon time. Also, note that, we can divide by $\beta$ and then allow it formally to vanish as done above for the PDE to obtain the finite difference equation for the Boussinesq model.

$$
\frac{\partial \tilde{\rho}_{i k}}{\partial t}+\tilde{F}_{\rho x i k}+\tilde{F}_{\rho z i k}+D_{i k}=0
$$

where

$$
\begin{aligned}
\tilde{F}_{\rho x i k}= & \frac{1}{4 \delta x}\left(\rho_{0, i+1, k}+\rho_{0, i-1, k}+\tilde{\rho}_{i+1, k}+\tilde{\rho}_{i-1, k}\right)\left(u_{i, k}-u_{i-1, k}\right) \\
& +\frac{1}{4 \delta x}\left(\rho_{0, i+1, k}-\rho_{0, i-1, k}+\tilde{\rho}_{i+1, k}-\tilde{\rho}_{i-1, k}\right)\left(u_{i, k}+u_{i-1, k}\right) \\
\tilde{F}_{\rho z i k}= & \frac{1}{4 \delta z}\left(\rho_{0, i, k+1}+\rho_{0, i, k+1}+\tilde{\rho}_{i, k+1}+\tilde{\rho}_{i, k-1}\right)\left(w_{i, k}-w_{i, k-1}\right) \\
& +\frac{1}{4 \delta z}\left(\rho_{0, i, k+1}-\rho_{0, i, k-1}+\tilde{\rho}_{i, k+1}-\tilde{\rho}_{i, k-1}\right)\left(w_{i, k}+w_{i, k-1}\right)
\end{aligned}
$$

and where $D_{i k}$ is the dimensionless form of $\Delta_{i k}$ divided by $\beta$,

$$
\left.D_{i k}=\left[\frac{\gamma-1}{\gamma} \frac{Q}{p_{0}}-\kappa \nabla^{2} \rho\right)\right]_{i k}
$$

Here $\kappa=K /\left(\rho C_{p}\right)$.

At boundaries, the density fluxes are determined by the no-inflow, nooutflow conditions. Also, we must specify adiabatic or cold-wall boundary conditions, which determine the temperature and hence the density (since the perturbation density is the negative of the perturbation temperature) in ficticious cells adjacent to the boundaries.

At $i=1$ :

$$
\begin{array}{r}
F_{\rho x 1 k}=\frac{1}{2 \delta x}\left(\rho_{0,2, k}+\tilde{\rho}_{2, k}\right) u_{2, k} \\
\tilde{\rho}_{0, k}= \pm \tilde{\rho}_{1, k}
\end{array}
$$


At $i=I$ :

$$
\begin{array}{r}
F_{\rho x I k}=-\frac{1}{2 \delta x}\left(\rho_{0, I-1, k}+\tilde{\rho}_{I-1, k}\right) u_{I-1, k} \\
\tilde{\rho}_{I+1, k}= \pm \tilde{\rho}_{I, k}
\end{array}
$$

At $k=1$ :

$$
\begin{array}{r}
F_{\rho z i 1}=\frac{1}{2 \delta z}\left(\rho_{0, i, 2}+\tilde{\rho}_{i, 2}\right) w_{i, 2} \\
\tilde{\rho}_{i, 0}= \pm \tilde{\rho}_{i, 1}
\end{array}
$$

At $k=K$ :

$$
\begin{array}{r}
F_{\rho z i K}=-\frac{1}{2 \delta z}\left(\rho_{0, i, K-1}+\tilde{\rho}_{i, K-1}\right) w_{i, K-1} \\
\tilde{\rho}_{i, K+1}= \pm \tilde{\rho}_{i, K}
\end{array}
$$

Here, the plus sign corresponds to adiabatic boundary conditions (BC) and the minus sign to cold wall $\mathrm{BC}$. Adiabatic $\mathrm{BC}$ imply zero derivative in the temperature (density) perturbation across the boundary whereas cold wall $\mathrm{BC}$ imply the temperature (density) perturbation is zero at the boundary.

Two different discretizations in time have been used. In each case, the discretizations of the time derivative has been chosen as a leap frog.

$$
\left(\frac{\partial \tilde{\rho}_{i k}}{\partial t}\right)^{n}=\frac{1}{2 \delta t}\left(\tilde{\rho}_{i k}^{n+1}-\tilde{\rho}_{i k}^{n-1}\right)
$$

In the first case, we use a lagged-diffusion temporal discretization for the dissipation.

$$
\begin{aligned}
\left(\nabla^{2} \rho\right)_{i k}^{n-1} & =\left(\nabla^{2}\left[\rho_{0}(z)+\tilde{\rho}\right]\right)_{i k}^{n-1} \\
=\frac{1}{\delta z^{2}}\left(\rho_{0, i, k+1}-2 \rho_{0, i k}+\rho_{0, i, k-1}\right) & -\left(\frac{1}{\delta x^{2}}+\frac{1}{\delta z^{2}}\right)\left(\tilde{\rho}_{i k}^{n-1}+\tilde{\rho}_{i k}^{n-1}\right) \\
+\frac{1}{\delta x^{2}}\left(\tilde{\rho}_{i+1, k}^{n-1}+\tilde{\rho}_{i-1, k}^{n-1}\right) & +\frac{1}{\delta z^{2}}\left(\tilde{\rho}_{i, k+1}^{n-1}+\tilde{\rho}_{i, k-1}^{n-1}\right)
\end{aligned}
$$

Define

$$
\begin{array}{r}
R_{\rho, i k}^{n}=-\tilde{F}_{\rho x, i k}^{n}-\tilde{F}_{\rho z, i k}^{n}-\frac{\gamma-1}{\gamma} \tilde{Q}_{i k}^{n}+\frac{\kappa}{\delta z^{2}}\left(\rho_{0, i, k+1}-2 \rho_{0, i k}+\rho_{0, i, k-1}\right) \\
-2\left(\frac{\kappa}{\delta z^{2}}+\frac{\kappa}{\delta z^{2}}\right)\left(\tilde{\rho}_{i, k}^{n-1}+\frac{\kappa}{\delta x^{2}}\left(\tilde{\rho}_{i+1, k}^{n-1}+\tilde{\rho}_{i-1, k}^{n-1}\right)+\frac{\kappa}{\delta z^{2}}\left(\tilde{\rho}_{i, k+1}^{n-1}+\tilde{\rho}_{i, k-1}^{n-1}\right)\right.
\end{array}
$$


We also define $\tilde{R}_{i, k}^{n}$ to be the same as $R_{i, k}^{n}$ but with all quantities defined at time level $n$. In this case, the temporal discretization for second-order time steps yields

$$
\tilde{\rho}_{i k}^{n+1}=2 \delta t R_{\rho, i k}^{n}+\tilde{\rho}_{i k}^{n-1}
$$

and for the first-order time steps (initially)

$$
\tilde{\rho}_{i k}^{n+1}=\delta t \tilde{R}_{\rho, i k}^{n}+\tilde{\rho}_{i k}^{n}
$$

On the other hand, when we use the DuFort-Frankel temporal discretization scheme

$$
\begin{aligned}
\left(\nabla^{2} \rho\right)_{i k}^{n} & =\left(\nabla^{2}\left[\rho_{0}(z)+\tilde{\rho}\right]\right)_{i k}^{n} \\
=\frac{1}{\delta z^{2}}\left(\rho_{0, i, k+1}-2 \rho_{0, i k}+\rho_{0, i, k-1}\right) & -\left(\frac{1}{\delta x^{2}}+\frac{1}{\delta z^{2}}\right)\left(\tilde{\rho}_{i k}^{n+1}+\tilde{\rho}_{i k}^{n-1}\right) \\
+\frac{1}{\delta x^{2}}\left(\tilde{\rho}_{i+1, k}^{n}+\tilde{\rho}_{i-1, k}^{n}\right) & +\frac{1}{\delta z^{2}}\left(\tilde{\rho}_{i, k+1}^{n}+\tilde{\rho}_{i, k-1}^{n}\right)
\end{aligned}
$$

In this case, we define

$$
\begin{aligned}
R_{\rho, i k}^{n}=-\tilde{F}_{\rho x, i k}^{n}-\tilde{F}_{\rho z, i k}^{n} & -\frac{\gamma-1}{\gamma} \tilde{Q}_{i k}^{n}+\frac{\kappa}{\delta z^{2}}\left(\rho_{0, i, k+1}-2 \rho_{0, i k}+\rho_{0, i, k-1}\right) \\
& +\frac{\kappa}{\delta x^{2}}\left(\tilde{\rho}_{i+1, k}^{n}+\tilde{\rho}_{i-1, k}^{n}\right)+\frac{\kappa}{\delta z^{2}}\left(\tilde{\rho}_{i, k+1}^{n}+\tilde{\rho}_{i, k-1}^{n}\right)
\end{aligned}
$$

The temporal distretization yields

$$
\frac{1}{2 \delta t}\left(\tilde{\rho}_{i k}^{n+1}-\tilde{\rho}_{i k}^{n-1}\right)=R_{\rho, i k}^{n}-\kappa\left(\frac{1}{\delta x^{2}}+\frac{1}{\delta z^{2}}\right)\left(\tilde{\rho}_{i k}^{n+1}+\tilde{\rho}_{i k}^{n-1}\right)
$$

We define

$$
r_{\rho}=2 \kappa \delta t\left(\frac{1}{\delta x^{2}}+\frac{1}{\delta z^{2}}\right)
$$

Then, for the second-order time steps

$$
\tilde{\rho}_{i k}^{n+1}=\left(1+r_{\rho}\right)^{-1}\left[2 \delta t R_{\rho, i k}^{n}+\left(1-r_{\rho}\right) \tilde{\rho}_{i k}^{n-1}\right]
$$

and for the first-order time steps (initially)

$$
\tilde{\rho}_{i k}^{n+1}=\delta t R_{\rho, i k}^{n}+\left(1-r_{\rho}\right) \tilde{\rho}_{i k}^{n}
$$


In these calculations, the following forms for the heat source have been used:

$$
\tilde{Q}_{i k}^{n}=f^{n} Q_{x, i} Q_{z, k}
$$

where $f^{n}$ is either

$$
f^{n}=A \sinh \left(\alpha t^{n}\right)
$$

or

$$
f^{n}=B \sin \left(\alpha t^{n}\right)
$$

for $0 \leq t \leq(\pi / \alpha)$, and $f^{n}=0$ for $t \geq \pi / \alpha$. Also,

$$
\begin{aligned}
& Q_{x, i}=\sqrt{\beta_{x} / \pi} \exp \left[-\beta_{x}\left(x_{i}-x_{c}\right)^{2}\right] \\
& Q_{z, k}=\sqrt{\beta_{z} / \pi} \exp \left[-\beta_{z}\left(z_{k}-z_{c}\right)^{2}\right]
\end{aligned}
$$

or

$$
Q_{z, k}=\lambda \exp \left[-\lambda z_{k}\right]
$$

\subsection{The Momentum Equations}

In the velocity equations, we use the following difference scheme:

$$
\begin{aligned}
& \frac{\partial u_{i k}}{\partial t}+F_{x i k}+\left(p_{i+1, k}-p_{i, k}\right) /(\delta x)-g_{x}\left(\rho_{i+1, k}+\rho_{i, k}\right) / 2=\nu\left(\nabla^{2} u\right)_{i k} \\
& \frac{\partial w_{i k}}{\partial t}+F_{z i k}+\left(p_{i, k+1}-p_{i, k}\right) /(\delta z)-g_{z}\left(\rho_{i, k+1}+\rho_{i, k}\right) / 2=\nu\left(\nabla^{2} w\right)_{i k}
\end{aligned}
$$

The velocity fluxes are defined as follows:

$$
\begin{gathered}
F_{x i k}=\left(q_{i+1, k}^{2}-q_{i k}^{2}\right) /(2 \delta x)+0.5\left(w_{\omega i k} \omega_{i k}+w_{\omega i, k-1} \omega_{i, k-1}\right) \\
F_{z i k}=\left(q_{i, k+1}^{2}-q_{i k}^{2}\right) /(2 \delta z)-0.5\left(u_{\omega i k} \omega_{i k}+u_{\omega i-1, k} \omega_{i-1, k}\right)
\end{gathered}
$$

where

$$
\begin{array}{r}
q_{i k}^{2}=0.25\left[\left(u_{i k}+u_{i-1, k}\right)^{2}+\left(w_{i k}+w_{i, k-1}\right)^{2}\right] \\
\omega_{i k}=\left(u_{i, k+1}-u_{i k}\right) /(\delta z)-\left(w_{i+1, k}-w_{i k}\right) /(\delta x) \\
w_{\omega i k}=0.5 *\left(w_{i k}+w_{i+1, k}\right) \\
u_{\omega i k}=0.5 *\left(u_{i k}+u_{i, k+1}\right)
\end{array}
$$


The temporal discretization is done exactly as for the density equation; we either used a lagged dissipation or a DuFort Frankel scheme. In both cases, the temporal derivatives are handled by a leap-frog discretization

$$
\begin{aligned}
\left(\frac{\partial u_{i k}}{\partial t}\right)^{n} & =\frac{1}{2 \delta t}\left(u_{i k}^{n+1}-u_{i k}^{n-1}\right) \\
\left(\frac{\partial w_{i k}}{\partial t}\right)^{n} & =\frac{1}{2 \delta t}\left(w_{i k}^{n+1}-w_{i k}^{n-1}\right)
\end{aligned}
$$

When we use a lagged dissipation method, we define the dissipation terms as follows:

$$
\begin{gathered}
\left(\nabla^{2} u\right)_{i k}^{n} \equiv \frac{1}{\delta x^{2}}\left(\dot{u}_{i+1, k}^{n}+u_{i-1, k}^{n}\right)+\frac{1}{\delta z^{2}}\left(u_{i, k+1}^{n}+u_{i, k-1}^{n}\right)-2\left(\frac{1}{\delta x^{2}}+\frac{1}{\delta z^{2}}\right) u_{i k}^{n} \\
\left(\nabla^{2} w\right)_{i k}^{n} \equiv \frac{1}{\delta x^{2}}\left(w_{i+1, k}^{n}+w_{i-1, k}^{n}\right)+\frac{1}{\delta z^{2}}\left(w_{i, k+1}^{n}+w_{i, k-1}^{n}\right)-2\left(\frac{1}{\delta x^{2}}+\frac{1}{\delta z^{2}}\right) w_{i k}^{n}
\end{gathered}
$$

And we define the right hand sides as follows:

$$
\begin{aligned}
& U_{i k}^{n} \equiv-F_{x i k}^{n}-\left(p_{i+1, k}^{n}-p_{i, k}^{n}\right) /(\delta x)+g_{x}\left(\rho_{i+1, k}^{n}+\rho_{i, k}^{n}\right) / 2+ \\
& +\nu\left(\nabla^{2} u\right)_{i k}^{n-1} \\
& W_{i k}^{n} \equiv-F_{z i k}^{n}-\left(p_{i, k+1}^{n}-p_{i, k}^{n}\right) /(\delta z)+g_{z}\left(\rho_{i, k+1}^{n}+\rho_{i, k}^{n}\right) / 2+ \\
& +\nu\left(\nabla^{2} w\right)_{i k}^{n-1}
\end{aligned}
$$

and

$$
\begin{aligned}
\tilde{U}_{i k}^{n} \equiv-F_{x i k}^{n}-\left(p_{i+1, k}^{n}-p_{i, k}^{n}\right) /(\delta x)+g_{x}\left(\rho_{i+1, k}^{n}\right. & \left.+\rho_{i, k}^{n}\right) / 2+ \\
& +\nu\left(\nabla^{2} u\right)_{i, k}^{n} \\
\tilde{W}_{i k}^{n} \equiv-F_{z i k}^{n}-\left(p_{i, k+1}^{n}-p_{i, k}^{n}\right) /(\delta z)+g_{z}\left(\rho_{i, k+1}^{n}\right. & \left.+\rho_{i, k}^{n}\right) / 2+ \\
& +\nu\left(\nabla^{2} w\right)_{i, k}^{n}
\end{aligned}
$$

For the second-order time steps using the lagged-dissipation scheme,

$$
\begin{gathered}
u_{i k}^{n+1}=2 \delta t U_{i k}^{n}+u_{i k}^{n-1} \\
w_{i k}^{n+1}=2 \delta t W_{i k}^{n}+w_{i k}^{n-1}
\end{gathered}
$$


and for the first-order time steps (initially) using the lagged dissipation

$$
\begin{gathered}
u_{i k}^{n+1}=\delta t \tilde{U}_{i k}^{n}+u_{i k}^{n} \\
w_{i k}^{n+1}=\delta t \tilde{W}_{i k}^{n}+w_{i k}^{n}
\end{gathered}
$$

In the DuFort-Frankel scheme,

$$
\begin{gathered}
\left(\nabla^{2} u\right)_{i k}^{n} \equiv \frac{1}{\delta x^{2}}\left(u_{i+1, k}^{n}+u_{i-1, k}^{n}\right)+\frac{1}{\delta z^{2}}\left(u_{i, k+1}^{n}+u_{i, k-1}^{n}\right)+\left(\frac{1}{\delta x^{2}}+\frac{1}{\delta z^{2}}\right)\left(u_{i k}^{n+1}+u_{i k}^{n-1}\right) \\
\left(\nabla^{2} w\right)_{i k}^{n} \equiv \frac{1}{\delta x^{2}}\left(w_{i+1, k}^{n}+w_{i-1, k}^{n}\right)+\frac{1}{\delta z^{2}}\left(w_{i, k+1}^{n}+w_{i, k-1}^{n}\right)+\left(\frac{1}{\delta x^{2}}+\frac{1}{\delta z^{2}}\right)\left(w_{i k}^{n+1}+w_{i k}^{n-1}\right)
\end{gathered}
$$

In this case, we define

$$
\begin{array}{r}
U_{i k}^{n} \equiv-F_{x i k}^{n}-\left(p_{i+1, k}^{n}-p_{i, k}^{n}\right) /(\delta x)+g_{x}\left(\rho_{i+1, k}^{n}+\rho_{i, k}^{n}\right) / 2+ \\
\nu\left[\frac{1}{\delta x^{2}}\left(u_{i+1, k}^{n}+u_{i-1, k}^{n}\right)+\frac{1}{\delta z^{2}}\left(u_{i, k+1}^{n}+u_{i, k-1}^{n}\right)\right] \\
W_{i k}^{n} \equiv-F_{z i k}^{n}-\left(p_{i, k+1}^{n}-p_{i, k}^{n}\right) /(\delta z)+g_{z}\left(\rho_{i, k+1}^{n}+\rho_{i, k}^{n}\right) / 2+ \\
\nu\left[\frac{1}{\delta x^{2}}\left(w_{i+1, k}^{n}+w_{i-1, k}^{n}\right)+\frac{1}{\delta z^{2}}\left(w_{i, k+1}^{n}+w_{i, k-1}^{n}\right)\right]
\end{array}
$$

We define

$$
r \equiv 2 \nu \delta t\left(\frac{1}{\delta x^{2}}+\frac{1}{\delta z^{2}}\right)
$$

Then, for the second-order time steps using the DuFort-Frankel scheme

$$
\begin{array}{r}
u_{i k}^{n+1}=(1+r)^{-1}\left[2 \delta t U_{i k}^{n}+(1-r) u_{i k}^{n-1}\right] \\
w_{i k}^{n+1}=(1+r)^{-1}\left[2 \delta t W_{i k}^{n}+(1-r) w_{i k}^{n-1}\right]
\end{array}
$$

and for the first-order time steps (initially) using DuFort-Frankel

$$
\begin{array}{r}
u_{i k}^{n+1}=\delta t U_{i k}^{n}+(1-r) u_{i k}^{n} \\
w_{i k}^{n+1}=\delta t W_{i k}^{n}+(1-r) w_{i k}^{n}
\end{array}
$$

\subsection{Stability}

The linear stability of each of the two schemes, the DuFort-Frankel or Fromm method and the lagged-dissipation method, has been checked for a single convection-diffusion equation. Derivation of the dispersion relation 
in each case is straightforward; however, analysis of the stability (location of the roots of the quadratic in the dispersion relation) in the Fromm method is not easy, and we have resorted to a theorem derived by Miller [16] referenced in the book of Peyret and Taylor [17] to guarantee the stability. Assessing stability and study of the dispersion relation for the lagged-dissipation method is much simpler.

We start with a single convection-diffusion equation

$$
\frac{\partial \theta}{\partial t}+U \frac{\partial \theta}{\partial x}+W \frac{\partial \theta}{\partial z}=\epsilon \nabla^{2} \theta
$$

where $\theta$ is the single dependent variable, $U$ and $V$ are the local linearized vector velocity components, $\epsilon$ is the dissipation coefficient and $\nabla^{2}$ is the Laplacian. define

$$
\theta\left(t^{n}, x_{j}, z_{k}\right) \equiv \theta_{j k}^{n}
$$

and let $x_{j}=j \delta x, z_{k}=k \delta z, t^{n}=n \delta t$.

First, consider the DuFort-Frankel or Fromm method, and difference the convection-diffusion equation as follows:

$$
\begin{array}{r}
\frac{\theta_{j, k}^{n+1}-\theta_{j, k}^{n-1}}{2 \delta t}+\frac{U}{2 \delta x}\left[\theta_{j+1, k}^{n}-\theta_{j-1, k}^{n}\right]+\frac{W}{2 \delta z}\left[\theta_{j, k+1}^{n}-\theta_{j, k-1}^{n}\right] \\
-\frac{\epsilon}{\delta z^{2}}\left[r^{2}\left(\theta_{j+1, k}^{n}+\theta_{j-1, k}^{n}\right)+\left(\theta_{j, k+1}^{n}+\theta_{j, k-1}^{n}\right)-\left(1+r^{2}\right)\left(\theta_{j, k}^{n+1}+\theta_{j, k}^{n-1}\right)\right]=0
\end{array}
$$

where $r \equiv \delta z / \delta x$. Now let

$$
\theta_{j, k}^{n}=A_{n} \exp \left[i \delta z\left(j /\left(r \lambda_{x}\right)+k / \lambda_{z}\right)\right]
$$

Then

$$
\begin{array}{r}
A_{n+1}\left[1+\frac{2 \delta \epsilon\left(1+r^{2}\right)}{\delta z^{2}}\right]-A_{n-1}\left[1-\frac{2 \delta \epsilon\left(1+r^{2}\right)}{\delta z^{2}}\right] \\
+i A_{n} \frac{2 Q \delta t}{\delta z}\left[\frac { U r } { Q } \operatorname { s i n } \left(\frac{\delta z}{r \lambda_{x}}+\frac{W}{Q} \sin \left(\frac{\delta z}{\lambda_{z}}\right]=\right.\right. \\
\frac{2 \delta t \epsilon}{\delta z^{2}}\left[r ^ { 2 } \operatorname { c o s } \left(\frac{\delta z}{r \lambda_{x}}+\cos \left(\frac{\delta z}{\lambda_{z}}\right]\right.\right.
\end{array}
$$

where $Q=\sqrt{U^{2}+W^{2}}$. 
Define

$$
\begin{array}{r}
d \equiv \frac{2 \delta t \epsilon\left(1+r^{2}\right)}{\delta z^{2}} \\
T \equiv \frac{2 Q \delta t}{\delta z}\left[\frac{U r}{Q} \sin \left(\frac{\delta z}{r \lambda_{x}}\right)+\frac{W}{Q} \sin \left(\frac{\delta z}{\lambda_{z}}\right)\right] \\
P \equiv \frac{2}{1+r^{2}}\left[r^{2} \cos \left(\frac{\delta z}{r \lambda_{x}}\right)+\cos \left(\frac{\delta z}{\lambda_{z}}\right)\right]
\end{array}
$$

then

$$
A_{n+1}(1+d)-A_{n-1}(1-d)-A_{n}(P d-2 i T)=0
$$

or, with $A_{n}=\theta^{n}$ and dividing through by $\theta^{n-1}$

$$
\theta^{2}(1+d)-\theta(P d-2 i T)-(1-d)=0
$$

Now, according to the theorem of Miller [16], if we define

$$
f(\theta)=\theta^{2}-\theta \frac{P d-2 i T}{1+d}-\frac{1-d}{1+d}
$$

and a polynomial, constructed from $f(\theta)$, as follows:

$$
\tilde{f}(\theta)=1-\theta \frac{P d+2 i T}{1+d}-\frac{1-d}{1+d} \theta^{2}
$$

then, we can construct a polynomial $f_{1}(\theta)$ of one degree lower than $f(\theta)$, which in this case is of first degree, as follows:

$$
\begin{array}{r}
f_{1}(\theta)=\frac{1}{\theta}[\tilde{f}(0) f(\theta)-f(0) \tilde{f}(\theta)] \\
=\theta\left[1-\left(\frac{1-d}{1+d}\right)^{2}\right]-\frac{1}{1+d}\left[P d\left(1+\frac{1-d}{1+d}\right)-2 i T\left(1-\frac{1-d}{1+d}\right)\right]
\end{array}
$$

The theorem, proved in Miller [16], states that, if $|\tilde{f}(0)|=1 \geq|f(0)|=$ $(1-d) /(1+d)$ and $\left|\theta_{1}\right| \leq 1$, where $\theta_{1}$ is the zero of $f_{1}\left(\theta_{1}\right)=0$, then both of the zeros of $f(\theta)=0$ are such that $|\theta| \leq 1$. Now

$$
\theta_{1}=P / 2-i T
$$


With the assumption that $r=1$, we can write $P$ and $T$ as follows:

$$
\begin{array}{r}
T=\frac{2 Q \delta t}{\delta z} \cos \alpha \cos \left[\frac{\delta z}{2}\left(\frac{1}{\lambda_{x}}-\frac{1}{\lambda_{z}}\right)\right] \sin \left[\frac{\delta z}{2}\left(\frac{1}{\lambda_{x}}+\frac{1}{\lambda_{z}}\right)\right] \\
P=2 \cos \left[\frac{\delta z}{2}\left(\frac{1}{\lambda_{x}}+\frac{1}{\lambda_{z}}\right)\right] \cos \left[\frac{\delta z}{2}\left(\frac{1}{\lambda_{x}}-\frac{1}{\lambda_{z}}\right)\right]
\end{array}
$$

If the Courant condition, $\frac{2 Q \delta t}{\delta z} \leq 1$, is satisfied, then $\left|\theta_{1}\right| \leq 1$, and Fromm's method is stable, a conclusion determined by Miller [16] and restated by Peyret and Taylor [17] for the one-dimensional convection-diffusion equation.

For the lagged-diffusion scheme, a similar analysis can be performed; we start with the difference form of the convection-diffusion equation:

$$
\begin{array}{r}
\frac{\theta_{j, k}^{n+1}-\theta_{j, k}^{n-1}}{2 \delta t}+\frac{U}{2 \delta x}\left[\theta_{j+1, k}^{n}-\theta_{j-1, k}^{n}\right]+\frac{W}{2 \delta z}\left[\theta_{j, k+1}^{n}-\theta_{j, k-1}^{n}\right] \\
-\frac{\epsilon}{\delta z^{2}}\left[r^{2}\left(\theta_{j+1, k}^{n-1}+\theta_{j-1, k}^{n-1}\right)+\left(\theta_{j, k+1}^{n-1}+\theta_{j, k-1}^{n-1}\right)-\left(1+r^{2}\right)\left(\theta_{j, k}^{n-1}+\theta_{j, k}^{n-1}\right)\right]=0
\end{array}
$$

The equation for the amplitude $A_{n}$ in this case becomes

$$
\begin{array}{r}
A_{n+1}\left[1+\frac{2 \delta \epsilon\left(1+r^{2}\right)}{\delta z^{2}}\right]-A_{n-1}\left[1-\frac{2 \delta \epsilon\left(1+r^{2}\right)}{\delta z^{2}}\right] \\
+i A_{n} \frac{2 Q \delta t}{\delta z}\left[\frac{U r}{Q} \sin \left(\frac{\delta z}{r \lambda_{x}}\right)+\frac{W}{Q} \sin \left(\frac{\delta z}{\lambda_{z}}\right)\right]= \\
A_{n-1} \frac{2 \delta t \epsilon}{\delta z^{2}}\left[r^{2} \cos \left(\frac{\delta z}{r \lambda_{x}}\right)+\cos \left(\frac{\delta z}{\lambda_{z}}\right)\right]
\end{array}
$$

or,

$$
A_{n+1}(1+d)-A_{n-1}(1-d)-A_{n-1} P d+A_{n} 2 i T=0
$$

where the same notion as for the Fromm method is used. Substitution of $A_{n}=\theta^{n}$ and division by $\theta^{n-1}$ yields the dispersion relation for the laggeddiffusion scheme

$$
\theta^{2}(1+d)+\theta 2 i T-[1-d(1-P)]=0
$$

The roots of this quadratic equation, the amplification factors for the scheme, are

$$
\theta=\frac{1}{1+d}\left[-i T \pm \sqrt{-T^{2}+(1+d)[1-d(1-P)]}\right]
$$


If the Courant condition, $\frac{2 Q \delta t}{\delta z} \leq 1$, is satisfied and $d=\frac{2 \delta t \epsilon\left(1+r^{2}\right)}{\delta z^{2}} \leq 1$, then, for both roots,

$$
|\theta|=\sqrt{\frac{1-d(1-P)}{1+d}} \leq 1
$$

since $|P| \leq 1$, and the scheme is stable.

\subsection{The Pressure Equation}

The incompressibility condition is

$$
\left(u_{i, k}-u_{i-1, k}\right) / \delta x+\left(w_{i, k}-w_{i, k-1}\right) / \delta z=0
$$

and this condition can be used to derive the linear algebraic equation system for the pressure. A way from boundaries, that is for $2 \leq i \leq I-1,2 \leq k \leq$ $K-1$, we have

$$
\begin{gathered}
\frac{p_{i+1, k}-2 p_{i, k}+p_{i-1, k}}{\delta^{2} x}+\frac{p_{i, k+1}-2 p_{i, k}+p_{i, k-1}}{\delta^{2} z}= \\
+\left(-F_{x, i, k}+F_{x, i-1, k}\right) / \delta x+\left(-F_{z, i, k}+F_{z, i, k-1}\right) / \delta z \\
\quad+g_{x}\left[-\left(\rho_{i+1, k}+\rho_{i, k}\right)+\left(\rho_{i, k}+\rho_{i-1, k}\right)\right] /(2 \delta x)+ \\
g_{z}\left[-\left(\rho_{i, k+1}+\rho_{i, k}\right)+\left(\rho_{i, k}+\rho_{i, k-1}\right)\right] /(2 \delta z)+T_{i k}
\end{gathered}
$$

Here the matrix $T_{i k}$ is due to the viscous terms ( $T$ is proportional to $\nu$ ), and, as above, the lack of a superscript implies that all quantities are to be evaluated at time level $n$.

At the boundaries, we have four edges and four corners at which to determine the equations. Along each of the edges and corners, we systematically write out the equations using the conditions normal to the boundary that there is no velocity nor velocity flux and the pressure is hydrostatic. For $1 \leq k \leq K$

$$
\begin{array}{r}
u_{0 k}=0 \\
u_{I k}=0 \\
F_{x, 0 k}=0 \\
F_{x, I k}=0 \\
\tilde{p}_{1 k}-\tilde{p}_{0 k}=\left(\delta x g_{x} / 2\right)\left(\tilde{\rho}_{1 k}+\tilde{\rho}_{0 k}\right) \\
\tilde{p}_{I+1, k}-\tilde{p}_{I k}=\left(\delta x g_{x} / 2\right)\left(\tilde{\rho}_{I+1, k}+\tilde{\rho}_{I k}\right)
\end{array}
$$


For $1 \leq i \leq I$

$$
\begin{aligned}
& w_{i 0}=0 \\
& w_{i K}=0 \\
& F_{z, i 0}=0 \\
& F_{z, i K}=0 \\
& \tilde{p}_{i 1}-\tilde{p}_{i 0}=\left(\delta z g_{z} / 2\right)\left(\tilde{\rho}_{i 1}+\tilde{\rho}_{i 0}\right) \\
& \tilde{p}_{i, K+1}-\tilde{p}_{i K}=\left(\delta z g_{z} / 2\right)\left(\tilde{\rho}_{i, K+1}+\tilde{\rho}_{i K}\right)
\end{aligned}
$$

Also, we must either apply free-slip or no-slip BC. For $0 \leq k \leq K$,

$$
\begin{array}{r}
w_{0, k}^{n}= \pm w_{1, k}^{n} \\
w_{I, k}^{n}= \pm w_{I-1, k}^{n}
\end{array}
$$

and for $0 \leq i \leq I$

$$
\begin{array}{r}
u_{i, 0}^{n}= \pm u_{i, 1}^{n} \\
u_{i, K}^{n}= \pm u_{i, K-1}^{n}
\end{array}
$$

where the plus sign corresponds to free-slip and the minus sign to no-slip $\mathrm{BC}$.

To account for tangential boundary conditions, we have included the matrix $T_{i k}^{n}$. The expression for the matrix $T_{i, j}$ is straightforward but tedious to derive. In the paragraphs below, we indicate a derivation for this matrix.

Define the divergence $D_{i, k}^{n}$ as follows

$$
D_{i, k}^{n} \equiv\left(u_{i, k}^{n}-u_{i-1, k}^{n}\right) / \delta x+\left(w_{i, k}^{n}-w_{i, k-1}^{n}\right) / \delta z
$$

If we impose the condition on all interior cells (at the center where the divergence is defined) that the divergence be zero after any time step, then $D_{i, k}^{n+1} \equiv 0$ translates into the condition that

$$
\left(U_{i, k}^{n}-U_{i-1, k}^{n}\right) / \delta x+\left(W_{i, k}^{n}-W_{i, k-1}^{n}\right) / \delta z=0
$$

where $U_{i, k}^{n}$ and $W_{i, k}^{n}$ are the right hand sides of the momentum equations defined above. Substitution for these expressions for the DuFort-Frankel 
scheme (the expressions for the lagged-dissipation scheme can be similarly derived) then yields

$$
\begin{array}{r}
\frac{p_{i+1, k}-2 p_{i, k}+p_{i-1, k}}{\delta^{2} x}++\frac{p_{i, k+1}-2 p_{i, k}+p_{i, k-1}}{\delta^{2} z}= \\
+\left(-F_{x, i, k}+F_{x, i-1, k}\right) / \delta x+\left(-F_{z, i, k}+F_{z, i, k-1}\right) / \delta z \\
+g_{x}\left[-\left(\rho_{i+1, k}+\rho_{i, k}\right)+\left(\rho_{i, k}+\rho_{i-1, k}\right)\right] /(2 \delta x)+ \\
g_{z}\left[-\left(\rho_{i, k+1}+\rho_{i, k}\right)+\left(\rho_{i, k}+\rho_{i, k-1}\right)\right] /(2 \delta z)- \\
+\frac{\nu}{\delta x}\left[\frac{1}{\delta x^{2}}\left(u_{i+1, k}^{n}+u_{i-1, k}^{n}\right)+\frac{1}{\delta z^{2}}\left(u_{i, k+1}^{n}+u_{i, k-1}^{n}\right)\right] \\
\left.-\frac{1}{\delta x^{2}}\left(u_{i, k}^{n}+u_{i-2, k}^{n}\right)-\frac{1}{\delta z^{2}}\left(u_{i-1, k+1}^{n}+u_{i-1, k-1}^{n}\right)\right] \\
+\frac{\nu}{\delta z}\left[\frac{1}{\delta x^{2}}\left(w_{i+1, k}^{n}+w_{i-1, k}^{n}\right)+\frac{1}{\delta z^{2}}\left(w_{i, k+1}^{n}+w_{i, k-1}^{n}\right)\right. \\
\left.-\frac{1}{\delta x^{2}}\left(w_{i+1, k-1}^{n}+w_{i-1, k-1}^{n}\right)-\frac{1}{\delta z^{2}}\left(w_{i, k}^{n}+w_{i, k-2}^{n}\right)\right]
\end{array}
$$

If we define

$$
\hat{D}_{i, k}^{n} \equiv\left(D_{i+1, k}^{n}+D_{i-1, k}^{n}\right) / \delta x^{2}+\left(D_{i, k+1}^{n}+D_{i, k-1}^{n}\right) / \delta z^{2}
$$

then, the pressure equation can be rewritten as

$$
\begin{gathered}
\frac{p_{i+1, k}-2 p_{i, k}+p_{i-1, k}}{\delta^{2} x}+\frac{p_{i, k+1}-2 p_{i, k}+p_{i, k-1}}{\delta^{2} z}= \\
+\left(-F_{x, i, k}+F_{x, i-1, k}\right) / \delta x+\left(-F_{z, i, k}+F_{z, i, k-1}\right) / \delta z \\
\quad+g_{x}\left[-\left(\rho_{i+1, k}+\rho_{i, k}\right)+\left(\rho_{i, k}+\rho_{i-1, k}\right)\right] /(2 \delta x)+ \\
g_{z}\left[-\left(\rho_{i, k+1}+\rho_{i, k}\right)+\left(\rho_{i, k}+\rho_{i, k-1}\right)\right] /(2 \delta z)+\nu \hat{D}_{i, k}^{n}
\end{gathered}
$$

Now, in cells away from boundaries, $2 \leq i \leq I-1$ and $2 \leq k \leq K-1$, by imposition, all of the $D_{i, k}^{n}=0$ and therefore all of the $\hat{D}_{i, k}^{n}=0$. Hence $T_{i, k}^{n}=\nu \hat{D}_{i, k}^{n}=0$. It is only in cells adjacent to the boundaries (and in corner cells) where the matrix $T_{i, k}^{n}$ will in general be nonzero. To derive an expression for this matrix, consider the cells adjacent to the left boundary, $i=1,2 \leq k \leq K-1$. Along this boundary, $u_{0, k}^{n}=0$ for all $n$ so that $U_{0, k}^{n}=0$ implies

$$
\left(p_{1, k}^{n}-p_{0, k}^{n}\right) /(\delta x)=-F_{x 0, k}^{n}+g_{x}\left(\rho_{1, k}^{n}+\rho_{0, k}^{n}\right) / 2+\nu\left[\frac{1}{\delta x^{2}}\left(u_{1, k}^{n}+u_{-1, k}^{n}\right)\right.
$$


Subtracting this equation from the general equation for pressure with $i=1$ yields the equation

$$
\begin{array}{r}
\frac{p_{2, k}-p_{1, k}}{\delta x^{2}}+\frac{p_{1, k+1}-2 p_{1, k}+p_{1, k-1}}{\delta z^{2}}=-F_{x, i, k} / \delta x-\left(F_{z, 1, k}-F_{z, 1, k-1}\right) / \delta z \\
-g_{x}\left(\rho_{2, k}+\rho_{1, k}\right) /(2 \delta x)+g_{z}\left[-\left(\rho_{1, k+1}+\rho_{1, k}\right)+\left(\rho_{1, k}+\rho_{1, k-1}\right)\right] /(2 \delta z) \\
+\nu \hat{D}_{1, k}^{n}+\frac{\nu}{\delta x^{3}}\left(u_{1, k}^{n}+u_{-1, k}^{n}\right)
\end{array}
$$

Now, we impose the condition that the divergence be zero in the ficticious cell outside of the left boundary (which we are free to do) $D_{0, k}^{n}=0$. This condition implies that

$$
u_{-1, k}^{n} / \delta x=\left(w_{0, k}^{n}-w_{0, k-1}^{n}\right) / \delta z
$$

Similarly, the condition that the divergence be zero in the cell adjacent to the left boundary $D_{1, k}^{n}=0$ implies that

$$
u_{1, k}^{n} / \delta x=-\left(w_{1, k}^{n}-w_{1, k-1}^{n}\right) / \delta z
$$

Now, for free-slip BC,

$$
w_{0, k}^{n}=w_{1, k}^{n}
$$

and, for no-slip BC,

$$
w_{0, k}^{n}=-w_{1, k}^{n}
$$

Using all of these relations implies that

$$
u_{-1, k}^{n}=\mp u_{1, k}^{n}
$$

where the minus sign applies for free-slip BC and the plus sign for no-slip BC. Using the imposed condition that $D_{0, k}^{n}=0$ implies that $\hat{D}_{1, k}^{n}=0$. Hence

$$
\nu \hat{D}_{1, k}^{n}+\frac{\nu}{\delta x^{3}}\left(u_{1, k}^{n}+u_{-1, k}^{n}\right)=\frac{\nu}{\delta x^{3}}\left(u_{1, k}^{n} \mp u_{1, k}^{n}\right)
$$

or, $T_{1, k}^{n}=0$ for free-slip BC, while $T_{1, k}^{n}=\left(2 \nu u_{1, k}^{n}\right) / \delta x^{3}$ for no-slip BC.

In a similar fashion, the matrix $T_{i, k}^{n}$ can be derived at all other cells adjacent to boundaries. In this fashion, we find that for free-slip BC, $T_{i k}^{n}=0$ for all $i$ and $k, 1 \leq i \leq I, 1 \leq k \leq K$. For no-slip $\mathrm{BC}$, the matrix is still zero at cells removed from the boundary; i.e., $T_{i k}^{n}=0$ for $2 \leq i \leq I-1,2 \leq$ $k \leq K-1$. At cells adjacent to the boundary, i.e., edge or corner cells, the matrix is specified below. 


\subsubsection{Edges}

For $k=1,2 \leq i \leq I-1$,

$$
\begin{array}{r}
\frac{p_{i+1,1}-2 p_{i, 1}+p_{i-1,1}}{\delta x^{2}}+\frac{p_{i, 2}-p_{i, 1}}{\delta z^{2}}= \\
+\left(-F_{x, i, 1}+F_{x, i-1,1}\right) / \delta x-F_{z, i, 1} / \delta z \\
+g_{x}\left[-\left(\rho_{i+1,1}+\rho_{i, 1}\right)+\left(\rho_{i, 1}+\rho_{i-1,1}\right)\right] /(2 \delta x) \\
-g_{z}\left(\rho_{i, 2}+\rho_{i, 1}\right) /(2 \delta z)+T_{i, 1}
\end{array}
$$

For $k=K, 2 \leq i \leq I-1$, we have

$$
\begin{array}{r}
\frac{p_{i+1, K}-2 p_{i, K}+p_{i-1, K}}{\delta x^{2}}+\frac{-p_{i, K}+p_{i, K-1}}{\delta z^{2}}= \\
+\left(-F_{x, i, K}+F_{x, i-1, K}\right) / \delta x+F_{z, i, K-1} / \delta z \\
+\left[-g_{x}\left(\rho_{i+1, K}+\rho_{i, K}\right)+g_{x}\left(\rho_{i, K}+\rho_{i-1, K}\right)\right] /(2 \delta x) \\
g_{z}\left(\rho_{i, K}+\rho_{i, K-1}\right) /(2 \delta z)+T_{i, K}
\end{array}
$$

Next do the vertical edges; $i=1,2 \leq k \leq K-1$.

$$
\begin{array}{r}
\frac{p_{2, k}-p_{1, k}}{\delta x^{2}}+\frac{p_{1, k+1}-2 p_{1, k}+p_{1, k-1}}{\delta z^{2}}= \\
-F_{x, 1, k} / \delta x+\left(-F_{z, 1, k}+F_{z, 1, k-1}\right) / \delta z \\
-g_{x}\left(\rho_{2, k}+\rho_{1, k}\right) /(2 \delta x)+\left[-g_{z}\left(\rho_{1, k+1}+\rho_{1, k}\right)+\right. \\
\left.g_{z}\left(\rho_{1, k}+\rho_{1, k-1}\right)\right] /(2 \delta z)+T_{1, k}
\end{array}
$$

For $i=I, 2 \leq k \leq K-1$.

$$
\begin{array}{r}
\frac{-p_{I, k}+p_{I-1, k}}{\delta x^{2}}+\frac{p_{I, k+1}-2 p_{I, k}+p_{I, k-1}}{\delta z^{2}}= \\
+F_{x, I-1, k} / \delta x+\left(-F_{z, I, k}+F_{z, I, k-1}\right) / \delta z \\
+g_{x}\left(\rho_{I, k}+\rho_{I-1, k}\right) /(2 \delta x)+\left[-g_{z}\left(\rho_{I, k+1}+\rho_{I, k}\right)+\right. \\
\left.g_{z}\left(\rho_{I, k}+\rho_{I, k-1}\right)\right] /(2 \delta z)+T_{I, k}
\end{array}
$$

\subsubsection{Corners}

Finally, do the corners. First, $i=1, k=1$

$$
\begin{array}{r}
\frac{p_{2,1}-p_{1,1}}{\delta x^{2}}+\frac{p_{1,2}-p_{1,1}}{\delta z^{2}}=-F_{x, 1,1} / \delta x \\
-F_{z, 1,1} / \delta z-g_{x}\left(\rho_{2,1}+\rho_{1,1}\right) /(2 \delta x) \\
-g_{z}\left(\rho_{1,2}+\rho_{1,1}\right) /(2 \delta z)+T_{1,1}
\end{array}
$$


Next, $i=I, k=1$.

$$
\begin{array}{r}
\frac{-p_{I, 1}+p_{I-1,1}}{\delta x^{2}}+\frac{p_{I, 2}-p_{I, 1}}{\delta z^{2}}=F_{x, I-1,1} / \delta x \\
-F_{z, I, 1} / \delta z+g_{x}\left(\rho_{I, 1}+\rho_{I-1,1}\right) /(2 \delta x) \\
-g_{z}\left(\rho_{I, 2}+\rho_{I, 1}\right) /(2 \delta z)+T_{I, 1}
\end{array}
$$

Next $i=1, k=K$.

$$
\begin{array}{r}
\frac{p_{2, K}-p_{1, K}}{\delta x^{2}}+\frac{-p_{1, K}+p_{1, K-1}}{\delta z^{2}}=-F_{x, 1, K} / \delta x \\
+F_{z, 1, K-1} / \delta z-g_{x}\left(\rho_{2, K}+\rho_{1, K}\right) /(2 \delta x) \\
+g_{z}\left(\rho_{1, K}+\rho_{1, K-1}\right) /(2 \delta z)+T_{1, K}
\end{array}
$$

For $i=I, k=K$.

$$
\begin{array}{r}
\frac{-p_{I, K}+p_{I-1, K}}{\delta x^{2}}+\frac{-p_{I, K}+p_{I, K-1}}{\delta z^{2}}=F_{x, I-1, K} / \delta x \\
+F_{z, I, K-1} / \delta z+g_{x}\left(\rho_{I, K}+\rho_{I-1, K}\right) /(2 \delta x) \\
+g_{z}\left(\rho_{I, K}+\rho_{I, K-1}\right) /(2 \delta z)+T_{I, K}
\end{array}
$$

Thus, the viscous matrix is defined as follows: For free-slip BC, for $1 \leq i \leq$ $I, 1 \leq k \leq K$

$$
T_{i, k}=0
$$

For no-slip BC, for $2 \leq i \leq I-1,2 \leq k \leq K-1$

$$
T_{i, k}=0
$$

for $2 \leq k \leq K-1$

$$
\begin{array}{r}
T_{1, k}=\frac{2 \nu}{\delta x^{3}} u_{1, k} \\
T_{I, k}=-\frac{2 \nu}{\delta x^{3}} u_{I-1, k}
\end{array}
$$

and for $2 \leq i \leq I-1$

$$
\begin{array}{r}
T_{i, 1}=\frac{2 \nu}{\delta z^{3}} w_{1, k} \\
T_{i, K}=-\frac{2 \nu}{\delta z^{3}} w_{i, K-1}
\end{array}
$$


Finally, at the corners

$$
\begin{array}{r}
T_{1,1}=\frac{2 \nu}{\delta z^{3}} w_{1,1}+\frac{2 \nu}{\delta x^{3}} u_{1,1} \\
T_{1, K}=-\frac{2 \nu}{\delta z^{3}} w_{1, K-1}+\frac{2 \nu}{\delta x^{3}} u_{1, K} \\
T_{I, 1}=-\frac{2 \nu}{\delta x^{3}} u_{I-1,1}+\frac{2 \nu}{\delta z^{3}} w_{I, 1} \\
T_{I, K}=-\frac{2 \nu}{\delta x^{3}} u_{I-1, K}-\frac{2 \nu}{\delta z^{3}} w_{I, K-1}
\end{array}
$$

Once again, no superscript implies quantities evaluated at time level $n$.

\section{$5 \quad$ Results}

In this section samples of results generated using a code implementing the algorithm described above are presented and discussed. The results represent some problems of interest to scientists concerned with enclosure fires. The aim of the computations is to investigate basic phenomena which occur in enclosure fires using current computational and graphical capabilities. The computations were performed on the Convex $\mathrm{C} 120$ in the Computational Combustion Facility, which is a joint facility of the Computing and Applied Mathematics Laboratory and Building and Fire Research Laboratory at NIST. The graphics were generated on four Silicon Graphics Personal Iris 4D20s, which are also part of this facility.

The scale of these computations is substantial. Runs have now been performed using almost one-half million grid points; they require nearly 50 megabytes and take up to 24 hours of CPU time. They are performed in 64 bit arithmetic. If we were to save any major fraction of the data generated, we would be overwhelmed. Therefore, a significant effort has been expended in trying to select only those data required to understand the phenomena being studied. We have concluded that Lagrangian particle tracking for transient phenomena is the most convenient method for saving and examining data from our computations, and this procedure is described below.

The flows are visualized by introducing Lagrangian particles into the heat source representing the fire. These particles are introduced by means 
of a random number generator which mimics the spatial distribution of heat in the source. Therefore, in general in the results shown, the particles are introduced according to a Gaussian distribution centered at the center of the heat source and with a half-width equal to that of the source. The particles are introduced at regular intervals of time, again mimicing the temporal dependence of the heat source, which is "turned on" rather quickly (on the dimensionless time of the computations) to a constant rate of heat release. Each set of particles is then allowed to move with the flow field so that one can see the movement of the "smoke and hot gases". The graphics are generated in color with the color representing the temperature of the particle. In this fashion, one can also discern where the flow is hot and where it has cooled by mixing and conduction. The color and the dynamic aspect of the graphics (a sequence of frames such as those exhibited below is displayed rapidly on the computer screen) enhance enormously the information which can be extracted from the computations.

The amount of computer memory, the CPU time and the data storage all increase as the resolution of the computations increases. If $I, K$ are the number of grid points in the $x$ and $y$ directions, then the memory requirements scale as $I K$. If we assume that $I=K$, then the CPU time scales as $K^{3}$ and the disk space scales as $K^{3}$ also if we retain data computed on the grid. If, on the other hand, we retain particle data, then the disk requirements can be decoupled from the grid size and are dependent on the number of Lagrangian particles introduced and the number of times these particle data are dumped. (Some additional time is required to compute the particle trajectories; however, as long as the number of particles introduced is small compared to the number of grid points, this additional CPU time is relatively small. In fact, for most computations we have done, this additional time amounts to only ten percent or less of the total CPU time.)

The resolution of the computation determines the Reynolds number of the flow which can be calculated, and this is a very important feature of the computations. Since the size of the Reynolds number for the 2-D flow scales as $K^{2}$, with the grids that we have been able to use, approximately one-half million grid cells, flows with a Reynolds number of nearly one-half million can in principle be computed. We have not yet computed flows with this large a value of the Reynolds number, primarily because we have been examining non-square geometries. However, we have computed results for flows with Reynolds numbers of up to $1 \times 10^{5}$. One comforting aspect of the 
algorithm and the code is that it will "bomb" if the Reynolds number is too large for the resolution of the grid. This feature, plus the fact that there are no adjustable parameters in the code, lead us to have great confidence in the predictive capability of the computations.

\subsection{Stratified Background Fluid}

A problem of some general interest is that of fire in an enclosure where the background fluid has been stratified [9], [10], [11], [12]. Stratification occurs naturally in any room that is relatively quiescent. In the summer, solar heating generally produces significant stratification, and during the winter, unless the stratification is disrupted, normal house heating also produces it. A small fire under this circumstance will have its induced flows significantly altered by the ambient stratification. Also, if a fire has started, produced a stratified upper layer in a room, temporarily died down, and then revives again, the plume dynamics will be affected by the stratification.

The first example is of a heat source (a line source in these 2-D calculations) at the floor of a rectangular enclosure. The aspect ratio of the room is 0.8 , the stratified layer occurs at 0.6 the height of the enclosure and the difference in density between the lower and upper layers is 5 times the characteristic density induced by the heat source. The heat source is centered on the floor, one-half unit (the length unit being the height of the room) from the left wall with a Gaussian half-width of approximately one-tenth unit. Four frames of a sequence are shown in Figure 1. The first frame shows the starting plume at 2.6 dimensionless time units. Note both the symmetry of the starting plume at this time and the detailed structure of the head of the starting plume. This structure is due to the high resolution of the computation, which has $600 \times 480$ grid cells, and has a Reynolds number of $1 \times 10^{5}$. The second frame in Figure 1 shows the plume at 3.2 time units. At this time, the plume has encountered the upper stratified layer; part of the plume penetrates the layer, but most of the plume is deflected laterally. Note the asymmetry which has begun to appear due to the fact that the heat source is not symmetrically placed in the room. In the third and fourth frames the plume continues to be deflected laterally because the upper layer is so strongly stratified. Again, note the asymmetry in the flow field.

Computations have also been carried out for other levels of stratification 
of the upper layer. As this stratification becomes stronger, the amount of penetration of the plume into the upper layer is reduced. On the other hand, as this stratification becomes smaller, more penetration occurs with the plume reaching the ceiling, and finally, with the stratification having little effect when it becomes very weak.

\subsection{Gravity Currents}

When lighter fluid is introduced at the top of an ambient fluid (or heavier fluid at the bottom of ambient fluid), the light fluid spreads over the ambient. The process by which this spreading takes place is called a gravity current. Gravity currents are well-known in the geophysical literature and have been studied both experimentally and theoretically for many years [13]. We have used our 2-D model, since gravity currents are primarily two-dimensional phenomena, to compute gravity currents with very high resolution and relatively high Reynolds numbers (up to $3 \times 10^{4}$ ). In Figure 2, we show several frames from a sequence of computed frames of a gravity current generated at the left of an enclosure and progressing to the right. The gravity current has the well-known characteristic "head" and progresses at a nearly uniform speed. The enclosure is 11 units long and one unit high, and has 1584 cells in the horizontal direction and 144 in the vertical (228,000 grid cells). The Reynolds for this computation is $1 \times 10^{4}$. From these computations, we can compare results with experimental measurements carried out elsewhere. First we can compare the velocity of the head of the gravity current and determine its variation with Reynolds number for example and later we may be able to make more detailed comparisons, comparing velocity or density profiles through the gravity current for example.

\subsection{The "Trench Effect"}

Fires in buildings involve the transport of heat and mass by gravity-induced or buoyant convection. Generally, this convection occurs in rectangular enclosures where the direction of gravity is parallel to the surfaces of the enclosure, the walls. However, under certain circumstances, such as a fire in a stair well or an escalator, the enclosure may be sloped relative to gravity. A very important example of a fire in a sloped enclosure was the devastat- 
ing fire in the King's Cross underground station in England in 1987, where there was significant loss of life as well as property damage. Numerical simulation of this fire uncovered an unexpected phenomenon which caused a very rapid spread of the fire and led to much of the devastation [14], [15]. This phenomenon was termed the "trench effect", and caused some controvery during investigations of the King's Cross fire in England. The phenomenon was ultimately confirmed by experiments and additional simulation, but transient aspects of the fire simulation are still of interest. We have examined this phenomenon using our 2-D code for Reynolds numbers of interest for enclosure fires.

In Figure 3, we present a sequence of frames from a 2-D, high-resolution computation for the flow generated in an enclosure by a heat source (fire) located on the floor one quarter of the length form the left wall. The resolution is $1296 \times 324$ (419,904 grid cells) in this $4 \times 1$ enclosure. The Reynolds number for this computation is $5 \times 10^{4}$, and free-slip and adiabatic boundary conditions have been imposed. The enclosure is inclined 35 degrees with respect to horizontal. In this figure, the plume rises, but is bent back toward the back wall. After the hot gases hit the ceiling, they progress both toward the back wall and up the ceiling toward the high end. However, the hot gases leaving the heat source are pinned along the floor and form a hot gas jet which progresses up along the floor, shedding hot gases near its front; this phenomenon we interpret as the "trench effect". These results were unexpected and were the reason that the "trench effect" caused so much controversary. 


\section{A Appendix: Particle Tracking Algorithm}

\section{A.1 Particle Injection}

The initial location of each particle is selected at random from a distribution function which is normal in the $\mathrm{x}$-direction with mean value equal to the location of the center of the heat source and variance equal to the mean width of the heat source. In the z-direction the distribution function is either normal with characteristics similar to that in the $\mathrm{x}$-direction or exponential. In all cases, the distribution functions mimic the spatial distribution of the heat source. Also, particles are injected at a rate that follows the rate of heat addition.

\section{A.2 Interpolation of the Fields at Particles Locations}

Take the 2-D case and let $x_{j}^{n}$ and $z_{j}^{n}$ be the coodinates of particle $j, 1 \leq$ $j \leq N_{p}$ at time $t^{n}$. Let the grid be our standard uniform grid,

$$
\begin{array}{cc}
x_{i}=i \delta x, & 0 \leq i \leq I \\
z_{k}=k \delta z, & 0 \leq k \leq K
\end{array}
$$

Let

$$
\begin{array}{r}
\mathcal{I}_{j} \equiv\left[x_{j}^{n} / \delta x+1 / 2\right] \\
\mathcal{K}_{j} \equiv\left[z_{j}^{n} / \delta z+1 / 2\right] \\
r_{j} \equiv x_{j}^{n} / \delta x-\left(\mathcal{I}_{j}-1 / 2\right) \\
s_{j} \equiv z_{j}^{n} / \delta z-\left(\mathcal{K}_{j}-1 / 2\right)
\end{array}
$$

and let

$$
\begin{array}{r}
\mathcal{I}_{j}^{\prime} \equiv\left[x_{j}^{n} / \delta x\right] \\
\mathcal{K}_{j}^{\prime} \equiv\left[z_{j}^{n} / \delta z\right] \\
r_{j}^{\prime} \equiv x_{j}^{n} / \delta x-\mathcal{I}_{j} \\
s_{j}^{\prime} \equiv z_{j}^{n} / \delta z-\mathcal{K}_{j}
\end{array}
$$

where $[. .$.$] is the integer part of the quantity in square brackets.$ 
Define the function $F$ as follows:

$F\left(A_{1,1}, A_{1,2}, A_{2,1}, A_{2,2}, r, s\right) \equiv A_{1,1}(1-r)(1-s)+A_{2,1} r(1-s)+A_{1,2}(1-r) s+A_{2,2} r s$ and let $\tilde{u}_{j}, \tilde{w}_{j}, \tilde{\rho}_{j}$ be the velocity components and the density respectively at the location of the $j$ th particle. Then by bilinear interpolation:

$$
\begin{gathered}
\tilde{u}_{j}^{n}=F\left(u_{\mathcal{I}_{j}^{\prime}+1, \mathcal{K}_{j}+1}^{n}, u_{\mathcal{I}_{j}^{\prime}+2, \mathcal{K}_{j}+1}^{n}, u_{I_{j}^{\prime}+1, \mathcal{K}_{j}+2}^{n}, u_{\mathcal{I}_{j}^{\prime}+2, \mathcal{K}_{j}+2}^{n}, r^{\prime}, s\right) \\
\tilde{w}_{j}^{n}=F\left(w_{\mathcal{I}_{j}+1, \mathcal{K}_{j}^{\prime}+1}^{n}, w_{\mathcal{I}_{j+2}, \mathcal{K}_{j}^{\prime}+1}^{n}, w_{\mathcal{I}_{j+1}, \mathcal{K}_{j}^{\prime}+2}^{n}, w_{\mathcal{I}_{j}+2, \mathcal{K}_{j}^{\prime}+2}^{n}, r, s^{\prime}\right) \\
\tilde{\rho}_{j}^{n}=F\left(\rho_{\mathcal{I}_{j}^{\prime}+1, \mathcal{K}_{j}^{\prime}+1}^{n}, \rho_{\mathcal{I}_{j}^{\prime}+2, \mathcal{K}_{j}^{\prime}+1}^{n}, \rho_{I_{j}^{\prime}+1, \mathcal{K}_{j}^{\prime}+2}^{n}, \rho_{I_{j}^{\prime}+2, \mathcal{K}_{j}^{\prime}+2}^{n}, r^{\prime}, s^{\prime}\right)
\end{gathered}
$$

\section{A.3 Time Advancement Scheme for Particles}

Now, each particle is advanced through the differential equation:

$$
\begin{aligned}
\frac{d x_{j}}{d t} & =u_{j}\left(x_{j}, z_{j}\right) \\
\frac{d z_{j}}{d t} & =w_{j}\left(x_{j}, z_{j}\right)
\end{aligned}
$$

The particles are advanced in time according to a second order RungeKutta time integration scheme as follows.

$$
\begin{gathered}
h_{x, j}^{n} \equiv \tilde{u}_{j}^{n}\left(x_{j}^{n}, z_{j}^{n}\right) \delta t \\
h_{z, j}^{n} \equiv \tilde{w}_{j}^{n}\left(x_{j}^{n}, z_{j}^{n}\right) \delta t
\end{gathered}
$$

Then, with

$$
\begin{array}{r}
\mathcal{I}_{j} \equiv\left[\left(x_{j}^{n}+h_{x, j}^{n}\right) / \delta x+1 / 2\right] \\
\mathcal{K}_{j} \equiv\left[\left(z_{j}^{n}+h_{z, j}^{n}\right) / \delta z+1 / 2\right] \\
r_{j} \equiv\left(x_{j}^{n}+h_{x, j}^{n}\right) / \delta x-\left(\mathcal{I}_{j}-1 / 2\right) \\
s_{j} \equiv\left(z_{j}^{n}+h_{z, j}^{n}\right) / \delta z-\left(\mathcal{K}_{j}-1 / 2\right)
\end{array}
$$

and let

$$
\begin{array}{r}
\mathcal{I}_{j}^{\prime} \equiv\left[\left(x_{j}^{n}+h_{x, j}^{n}\right) / \delta x\right] \\
\mathcal{K}_{j}^{\prime} \equiv\left[\left(z_{j}^{n}+h_{z, j}^{n}\right) / \delta z\right] \\
r_{j}^{\prime} \equiv\left(x_{j}^{n}+h_{x, j}^{n}\right) / \delta x-\mathcal{I}_{j} \\
s_{j}^{\prime} \equiv\left(z_{j}^{n}+h_{z, j}^{n}\right) / \delta z-\mathcal{K}_{j}
\end{array}
$$


The new extrapolated velocity components at location $x_{j}^{n}+h_{x, j}^{n}, z_{j}^{n}+h_{z, j}^{n}$ are

$$
\begin{array}{r}
\tilde{u}_{j}^{n}=F\left(u_{\mathcal{I}_{j}^{\prime}+1, \mathcal{K}_{j}+1}^{n}, u_{\mathcal{I}_{j}^{\prime}+2, \mathcal{K}_{j}+1}^{n}, u_{\mathcal{I}_{j}^{\prime}+1, \mathcal{K}_{j}+2}^{n}, u_{\mathcal{I}_{j}^{\prime}+2, \mathcal{K}_{j}+2}^{n}, r^{\prime}, s\right) \\
\tilde{w}_{j}^{n}=F\left(w_{\mathcal{I}_{j}+1, \mathcal{K}_{j}^{\prime}+1}^{n}, w_{\mathcal{I}_{j}+2, \mathcal{K}_{j}^{\prime}+1}^{n}, w_{\mathcal{I}_{j}+1, \mathcal{K}_{j}^{\prime}+2}^{n}, w_{\mathcal{I}_{j}+2, \mathcal{K}_{j}^{\prime}+2}^{n}, r, s^{\prime}\right)
\end{array}
$$

Finally, the location of the particle at time level $n+1$ is

$$
\begin{aligned}
& x_{j}^{n+1}=x_{j}^{n}+0.5\left(\tilde{u}_{j}^{n} \delta t+h_{x, j}^{n}\right) \\
& z_{j}^{n+1}=z_{j}^{n}+0.5\left(\tilde{w}_{j}^{n} \delta t+h_{z, j}^{n}\right)
\end{aligned}
$$

The accuracy of this particle-tracking routine was tested using a steadystate flow with vorticity in a rectangular enclosure. This flow field was originally derived to test the algorithms that integrate the fluid equations and is described in [18]. In the flow, particles follow streamlines which close upon themselves, a sensitive test of the quality of the particle-tracking integration scheme.

\section{References}

[1] Rehm, R.G. and H.R. Baum, "The Equations of Motion for Thermally Driven, Buoyant Flows", Journal of Research of the NBS, 83, pp 297308, May-June 1978.

[2] Baum, H.R., R.G. Rehm, P.D. Barnett and D.M. Corley, "Finite Difference Calculations of Buoyant Convection in an Enclosure", SIAM J. Sci. Stat. Comput., 4, pp 117-135, March 1983.

[3] Baum, H.R. and Rehm, R.G., " Finite Difference Solutions for Internal Waves in Enclosures", SIAM J. Sci. Stat. Comput., 5, No. 4, pp. 958977 (1984).

[4] Rehm, R.G., P.D. Barnett, H.R. Baum and D.M. Corley, "Finite Difference Calculations Of Buoyant Convection in an Enclosure: Verification of the Nonlinear Algorithm", Applied Numerical Mathematics, 1, pp 515-529, North-Holland, 1985. 
[5] Baum, H.R., Rehm, R.G. and Mulholland, G.W., "Computation of Fire Induced Flow and Smoke Coagulation", Nineteenth Symposium (International) on Combustion/ The Combustion Institute, pp 921931, Pittsburgh, PA, 1982.

[6] Baum, H.R. and R.G. Rehm, "Calculations of Three-Dimensional Buoyant Plumes in Enclosures", Combustion Science and Technology, 40, pp 55-77, Gordon and Breach Science Publishers, 1984.

[7] Rehm, R.G., Baum, H.R., Lozier, D.W. and Corley, D.M., "A Model of Three-Dimensional Buoyant Convection Induced by a Room Fire", First National Fluid Dynamics Congress, joint conference sponsored by the A.I.A.A., A.S.M.E., A.P.S. and S.I.A.M., July 24-28, 1988, Cincinnati, Ohio.

[8] Baum, H.R. and Rehm, R.G., "Transient Combustion in a Turbulent Fire", 9th Joint Meeting of the U.S. Japan Natural Resources (UJNR) Panel on Fire Research and Safety, Boston, MA, May, 1987.

[9] Mulholland, G., Handa, T., Sugawa, O., and Yamamoto, H., Fire Science and Technology, 1, p.1 (1981).

[10] Cooper, L.Y., Harkelroad, M., Quintiere, J. and Rinkinen, W., J. Heat Transfer, 104, p. 741 (1982).

[11] Cooper, L.Y., "A Buoyant Source in the Lower of Two, Homogeneous, Stably Stratified Layers", Twentieth Symposium (International) on Combustion, The Combustion Institute, pp 1567-1573 (1984).

[12] Kulkarni, A.N., Hwang, J.J., and Murphy, F., "Fire and Fire-Induced Flows in a Stratified Atmosphere" National Bureau of Standards Report NBS-GCR-88-548, pp 36-41, June 1988.

[13] Simpson, J.E. Gravity Currents in the Environment and the Laboratory, Halsted Press: a division of John Wiley \& Sons, New York, 1987.

[14] Simcox, S., Wilkes, N.S. and Jones, I.P., "Fire at King's Cross Underground Station, 18th November 1987: Numerical Simulation of the Buoyant Flow and Heat Transfer", Harwell Report AERE-G 4677, May 1988. 
[15] Cox, G., Chitty, R. and Kumar, S., "Fire Modeling and the King's Cross Fire Investigation", Letter to the Editor, Fire Safety Journal, 15 pp. 103-106, 1989.

[16] Miller, J.J.H., "On the Location of Zeros of Certain Classes of Polynomials with Applications to Numerical Analysis", J. Inst. Maths. Applics., 8, 397-406, 1971.

[17] Peyret, R. and Taylor, T.D., Computational Methods for Fluid Flow, Springer-Verlag, New York, 1983.

[18] Rehm, R.G., Baum, H.R. and Barnett, P.D., "Buoyant Convection Computed in a Vorticity, Stream-Function Formulation," Journal of Research of the NBS, 87, No. 2, March-April 1982, pp. 165-185. 


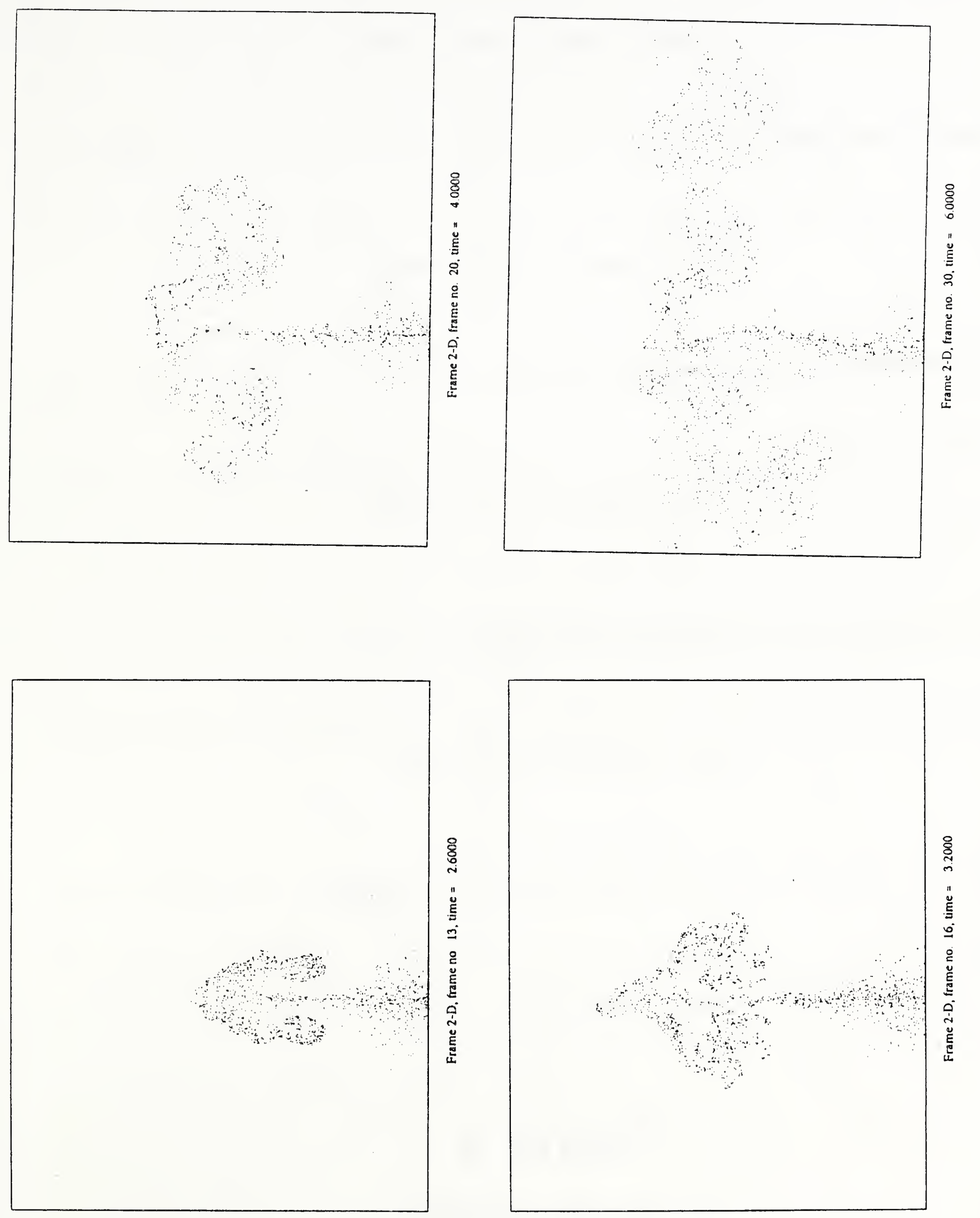

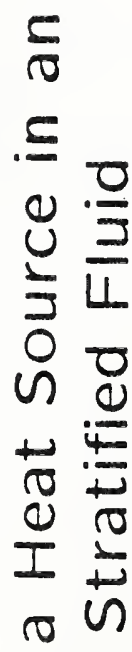

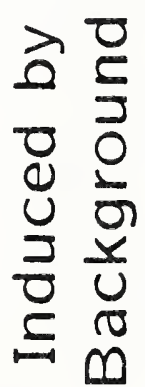

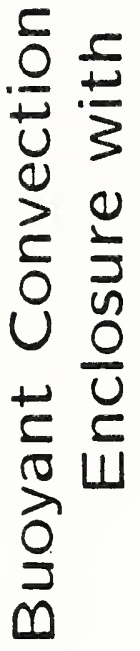

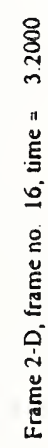

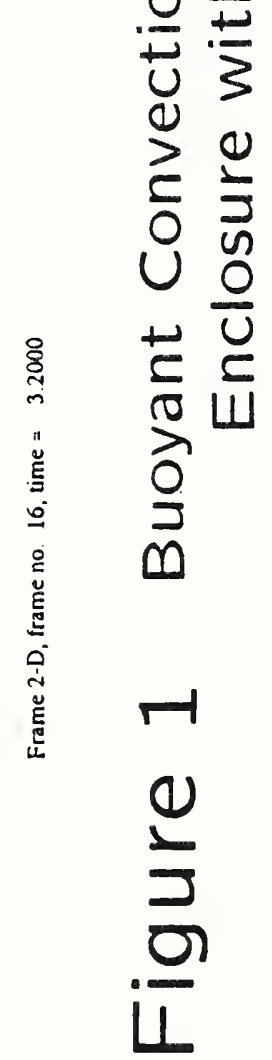


Frame 2-D, frame no. 5 , time $=5.0000$

Frame 2-D, frame no. 10 , time $=10.0000$

Frame 2-D, frame no. 20 , time $=20.0000$

Frame 2-D, frame no. 30, time $=30.0000$

:4.

Frame 2-D, frame no. 40 , time $=40.0000$

\section{Figure 2}

A Gravity Current 

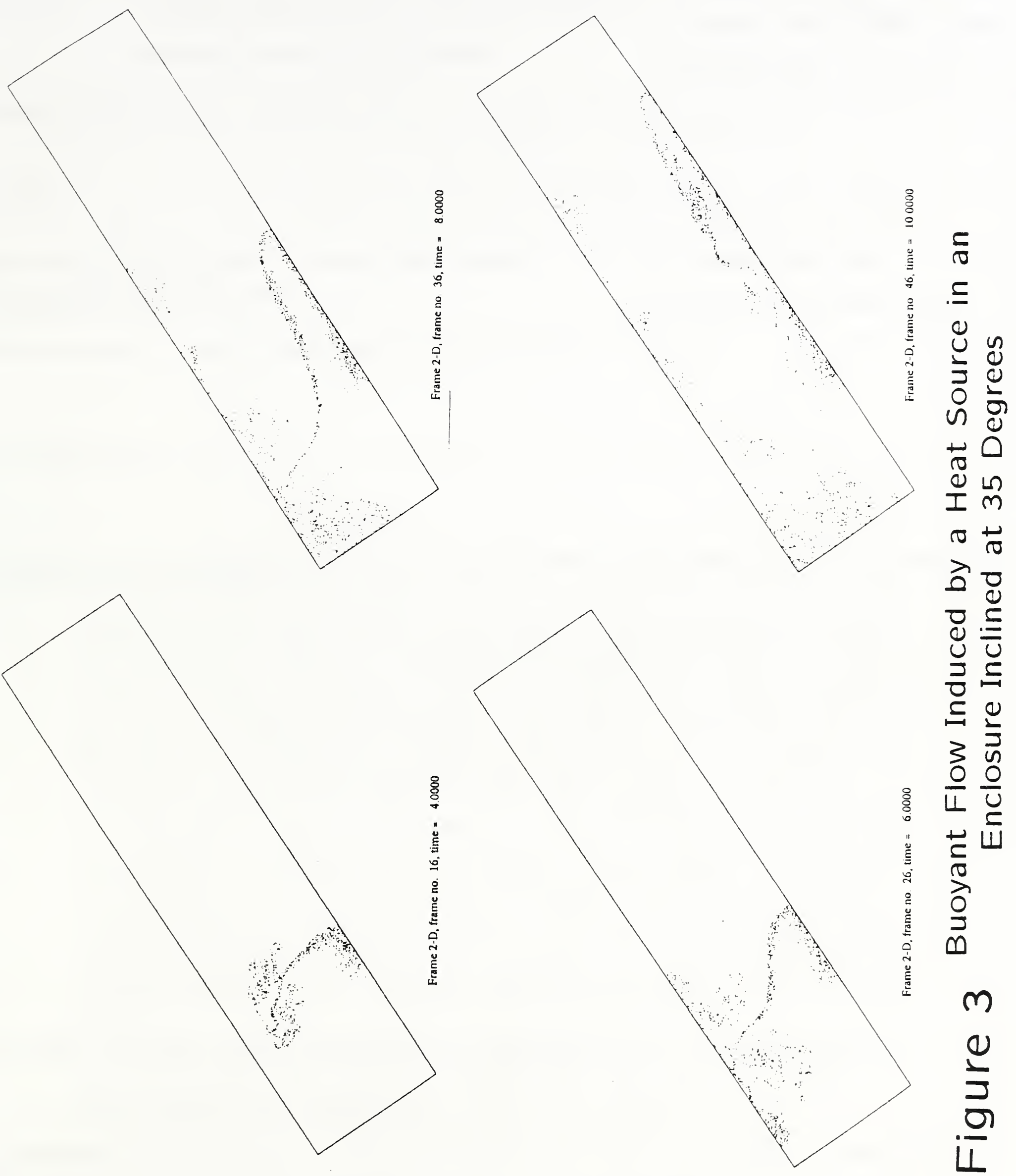



\begin{tabular}{|c|c|}
\hline \multirow[t]{3}{*}{$\begin{array}{l}\text { U.S. DEPARTMENT OF COMMERCE } \\
\text { NATIONAL INSTITUTE OF STANDARDS AND TECHNOLOGY }\end{array}$} & $\begin{array}{r}\text { 1. PUBLCATION OR REPORT NUMBER } \\
\text { NISTIR } 4540\end{array}$ \\
\hline & 2. PERFORMINQ ORGANIZATION REPORT NUMBER \\
\hline & 3. PUBLICATION DATE \\
\hline \multicolumn{2}{|c|}{$\begin{array}{l}\text { 4. TITLEAND SUBTITLE } \\
\text { A Boussinesq Algorithm for Enclosed Buoyant Convection in Two Dimensions }\end{array}$} \\
\hline \multicolumn{2}{|l|}{ 5. AUTHOR(S) } \\
\hline R.G. Rehm, 710; H.C. Tang, 718; H.R. Baum, 752; and J.S. Sims & 718 Corley, D.M., 752; \\
\hline \multirow{2}{*}{$\begin{array}{l}\text { 6. PERFORMINQ ORGANIZATION (IF JOINT OR OTHER THAN NIST, SEE INSTRUCTIONS) } \\
\text { U.S. DEPARTMENT OF COMMERCE } \\
\text { NATIONAL INSTITUTE OF STANDARDS AND TECHNOLOQY } \\
\text { GAITHERSBURQ, MD 20899 }\end{array}$} & 7. CONTRACT/GRANT NUMBER \\
\hline & 8. TYPE OF REPORT AND PERIOD COVERED \\
\hline
\end{tabular}

9. SPONSORINQ ORGANIZATION NAME AND COMPLETE ADDRESS (STREET, CITY, STATE, ZIP)

10. SUPPLEMENTARY NOTES

11. ABSTRACT (A 2OO-WORD OR LESS FACTUAL SUMMARY OF MOST SIGNIFICANT INFORMATION. IF DOCUMENT INCLUDES A SIONIFICANT BIBLOORAPHY OR UTERATURE SURVEY, MENTION IT HERE.)

Approximate equations for a Boussinesq model with viscous dissipation and thermal conduction describing buoyant convection driven by a heat source in a rectangular enclosure are derived. The finite difference algorithm for computing transient solutions in two dimensions to these equations is presented. The algorithm allows the enclosure fluid to be stratified in a direction parallel to the enclosure walls initially, or for gravity to have an arbitrary direction relative to the enclosure (but with no initial stratification). Computational results of transient, twodimensional buoyant convection for very high resolution are presented. The hydrodynamics is directly based on the time-dependent Navier-Stokes equations; the model is valid in the Boussinesq approximation. No turbulence model or other empirical parameters are introduced. There is no inflow or outflow at boundaries; this assumption, although rather restrictive, allows the mathematical problem to be properly formulated so that no other empiricism is introduced by specification of the algorithmic boundary conditions. A finite-difference scheme secondorder in space and first-order in time is used to integrate the evolution equations, and an elliptic solver is used to solve the pressure equation. The algorithms have been verified by comparisons with exact solutions to the equations in simple, special cases, and predictions of the overall model when the viscosity and thermal conductivity are zero have been compared with experimental results. The use of Lagrangian particle tracking allows one to visualize the flow patterns.

12. KEY WORDS (6 TO 12 ENTRIES; ALPHABETICAL ORDER; CAPITALZE ONLY PROPER NAMES; AND SEPARATE KEY WORDS BY SEMICOLONS)

Algorithm; Boussinesq Flow; Computational Fluid Dynamics; Fire-driven Flows; Gravity Currents; Stratified Flow; Trench Effect 


